\title{
Review: calcium antagonists lead to a higher risk for myocardial infarction, congestive heart failure, and major cardiovascular events
}

Pahor M, Psaty BM, Alderman MH, et al. Health outcomes associated with calcium antagonists compared with other first-line antihypertensive therapies: a meta-analysis of randomised controlled trials. Lancet 2000 Dec 9;356:1949-54.

QUESTION: In patients with hypertension, are calcium antagonists (CAs) as first line treatment superior, equal, or inferior to other antihypertensive drugs for reducing major cardiovascular events?

\section{Data sources}

Studies were identified by searching Medline up to September 2000, the US National Library of Medicine clinical trials database, and the Cochrane Library; by reviewing reference lists of identified articles; and by contacting colleagues.

\section{Study selection}

Studies were selected if they were randomised controlled trials published in peer reviewed journals that included $>100$ patients with hypertension who were allocated to first line treatment with a CA or other antihypertensive agent and who were followed for $\geqslant 2$ years for the assessment of cardiovascular events.

336713858 .

Calcium antagonists (CAs) $v$ other antihypertensive agents in hypertension*

\begin{tabular}{|c|c|c|c|c|}
\hline \multirow[b]{2}{*}{ Outcomes } & \multicolumn{2}{|c|}{ Weighted event rates } & \multirow[b]{2}{*}{ RRI (95\% CI) } & \multirow[b]{2}{*}{ NNH (Cl) } \\
\hline & CAs & Other agents & & \\
\hline MI & $4.5 \%$ & $3.6 \%$ & $25 \%$ (11 to 41$)$ & 110 (67 to 259$)$ \\
\hline Congestive heart failure & $3.3 \%$ & $2.7 \%$ & $24 \%(7$ to 44$)$ & 152 (83 to 538$)$ \\
\hline Major cardiovascular events & $14.2 \%$ & $13.1 \%$ & $24 \%(7$ to 44$)$ & 90 (51 to 442$)$ \\
\hline All-cause mortality & $8.3 \%$ & $8.1 \%$ & $3 \%(-6$ to 12$)$ & Not significant \\
\hline & CAs & Other agents & RRR (Cl) & NNT (Cl) \\
\hline Stroke & $4.5 \%$ & $5.0 \%$ & $10 \%(-2$ to 19$)$ & Not significant \\
\hline
\end{tabular}

* Follow up ranged from 2-7 years. $\mathrm{MI}=$ myocardial infarction. Other abbreviations defined in glossary; RRI, RRR, $\mathrm{NNH}, \mathrm{NNT}$, and $\mathrm{Cl}$ calculated from data in article.

\section{Data extraction}

Data were extracted on drug type and dose, patient characteristics, and main outcomes. Summary data were pooled.

\section{Main results}

9 trials involving 27743 patients with about 120000 person years of follow up met the selection criteria. CAs and other antihypertensive agents led to similar reductions in blood pressure. Patients assigned to diuretics, $\beta$ blockers, angiotensin converting enzyme (ACE) inhibitors, or clonidine $(n=15044)$ had a lower risk for myocardial infarction (MI), congestive heart failure, and major cardiovascular events than those allocated to CAs ( $\mathrm{n}=12$ 699) (table). No statistically significant differences were found for stroke or all cause mortality (table).

\section{Conclusion}

In patients with hypertension, initial treatment with calcium antagonists leads to higher risks for myocardial infarction, congestive heart failure, and major cardiovascular events than treatment with other antihypertensive agents.

\section{COMMENTARY}

Bottom line interpretations of these 2 meta-analyses will vary depending on previous beliefs. For methodological purists and fans of CAs, the data neither prove nor refute the choice of CAs as first line agents for adults with hypertension or those at high risk for cardiovascular disease. For those of us who are already wary of using CAs as a first line treatment, the data support our practice of avoiding these agents. Many of us, regardless of our previous beliefs, may now question whether we should either begin reserving or using CAs specifically for people who have high risk for stroke. Such a decision would be premature for at least 2 reasons. Firstly, we cannot reliably determine whether an individual is likely to have a stroke and no cardiac outcomes. Secondly, the important meta-analyses summarised here are limited and complicated.

The meta-analyses have limited power to precisely assess stroke and cardiac outcomes because few large, long term trials exist that directly compare the effects of different antihypertensive agents. They are complicated because they combine data from trials characterised by either discontinuation of initial antihypertensive drug treatments or the addition of second and third antihypertensive agents. They make comparisons by grouping all drugs within a class together (eg, CAs) as well as by grouping drugs across classes together (eg, diuretics and $\beta$ blockers). This method is dicey because all antihypertensive drugs have actions other than that of lowering blood pressure, and mechanisms by which a drug lowers blood pressure probably have effects independent of the blood pressure lowering effect. ${ }^{1}$ Moreover, mechanisms of action and effects may vary among antihypertensive drugs within classes as well as across classes. 


\section{Review: ACE inhibitors, calcium antagonists, and more intensive blood pressure lowering strategies reduce cardiovascular events}

\begin{abstract}
Blood Pressure Lowering Treatment Trialists'Collaboration. Effects of ACE inhibitors, calcium antagonists, and other blood-pressure-lowering drugs: results of prospectively designed overviews of randomised trials. Lancet 2000 Dec 9;356:1955-64.

QUESTION: In patients with hypertension, diabetes mellitus, coronary artery disease (CAD), or renal disease, what are the effects of angiotensin converting enzyme (ACE) inhibitors, calcium antagonists (CAs), and other blood pressure lowering drugs on mortality and major cardiovascular morbidity?
\end{abstract}

\section{Data sources}

In 1995, a registry was established of all large scale trials that were in progress or in advanced stages of planning.

\section{Study selection}

Studies were selected if there was a randomised comparison between patients allocated to a blood pressure lowering drug and placebo or to another active antihypertensive agent or to a blood pressure lowering strategy that had a different intensity. Trials had to have a minimum of 1000 patient years of follow up planned and must not have been published before July 1995 .

\section{Data extraction}

Data were extracted on patient characteristics, discontinuation rates, blood pressure, and major outcomes. Individual patient data or summary data were combined.

\section{Main results}

Data from 15 trials (74 696 patients, mean age 62 y, 53\% men) were included. Combining data from 4 placebo controlled trials of ACE inhibitors showed that active treatment led to relative reductions of $20 \%$ to $30 \%$ in stroke, CAD, and major cardiovascular events. Combining data from 2 placebo controlled trials of CAs showed that active treatment led to relative reductions of $28 \%$ to $39 \%$ in stroke and major cardiovascular events. Combining data from 3 trials of blood pressure lowering regimens of different intensity showed that more intensive strategies led to greater relative reductions of $15 \%$ to $20 \%$ in stroke, CAD, and major cardiovascular events. Combining data from 3 trials that compared ACE inhibitors with diuretics or $\beta$ blockers showed no differences between the agents for any outcome. Combining data from 5 trials that compared CAs with diuretics or $\beta$ blockers showed a slightly lower risk of stroke and a slightly higher risk of CAD with CAs (table).

\section{Conclusions}

In patients with hypertension, diabetes mellitus, coronary artery disease, or renal disease, angiotensin converting enzyme inhibitors and calcium antagonists are more beneficial than placebo. Calcium antagonists lead to a lower risk for stroke but to a slightly higher risk for coronary artery disease than diuretics or $\beta$ blockers.
Sources of funding:

National Health and

Medical Research

Council of Australia;

Medical Foundation of

the University of

Sydney; National Heart

Foundation of

Australia; Health

Research Council of

New Zealand; British

Heart Foundation;

International Society of

Hypertension;

AstraZeneca;Bayer A

$G ;$ Bristol

Myers-Squibb; Glaxo

Wellcome SpA; Hoechst

A G;Merck;Pfizer;

Searle and Servier.

For correspondence:

Dr B Neal, Blood

Pressure Lowering

Treatment Trialists

Collaboration, Institute for International

Health, University of

Sydney, PO Box 576,

Newtown, Sydney, New

South Wales 2042,

Australia. Fax +61 2

93510064.

\section{COMMENTARY-continued from previous page}

The reviews include trials that involved various groups of patients with different risks for cardiovascular disease. Pathways that lead people with diabetes, renal disease, hypertension, or CAD to experience cardiovascular outcomes may differ. Antihypertensive agents may have specific effects on causal pathways unique to particular groups of patients. Generalising the results of meta-analyses that are based on few trials is potentially misleading: 1 or 2 large trials that tested a specific drug in a particular population could drive the results. The determinants of cause specific outcomes, such as stroke and CAD, probably differ, even among people who share common risk factors (eg, patients with hypertension). Although such drugs as CAs may differentially affect pathways that lead to strokes from those that lead to $\mathrm{CAD}$, meta-analyses based on few trials cannot easily disentangle specific from class effects of antihypertensive drugs. Finally, the reviews are limited because they do not address the side effects and costs of different drugs.

Regardless, both meta-analyses are important summaries of accumulating evidence. Some diuretics, $\beta$ blockers, ACE inhibitors, and CAs have proven morbidity and mortality benefits for some people with hypertension or some who are at high risk for cardiovascular disease. Knowledge about the relative benefits and harms of the different drugs in particular groups of patients is murky but will probably become clearer in the next 5 years as larger trials that directly compare antihypertensive agents become available.

Cynthia Mulrow, MD, MSc Audie L Murphy Veterans Hospital San Antonio, Texas, USA

1 Wright JM, Lee CH, Chambers GK. Systematic review of antihypertensive therapies: does the evidence assist in choosing a first-line drug? CMAJ 1999;161:25-32. 